\title{
INTEGER LINEAR MODELS WITH A POLYNOMIAL NUMBER OF VARIABLES AND CONSTRAINTS FOR SOME CLASSICAL COMBINATORIAL OPTIMIZATION PROBLEMS
}

\author{
Nelson Maculan * \\ Rio de Janeiro - RJ \\ maculan@cos.ufrj.br \\ Gérard Plateau \\ LIPN - Institut Galilée \\ Université Paris 13 \\ Villetaneuse - France \\ gp@lipn.univ-paris13.fr
}

COPPE - Prog. de Eng. de Sistemas e Computação

Universidade Federal do Rio de Janeiro

\author{
Abdel Lisser \\ LRI - Université Paris Sud \\ Orsay Cedex - France \\ $\underline{\text { abdel.lisser@1ri.fr }}$ \\ * Corresponding author/autor para quem as correspondências devem ser encaminhadas \\ Recebido em 04/2002, aceito em 11/2002 após 1 revisão
}

\begin{abstract}
We present integer linear models with a polynomial number of variables and constraints for combinatorial optimization problems in graphs: optimum elementary cycles, optimum elementary paths and optimum tree problems.
\end{abstract}

Keywords: modeling combinatorial optimization problems; optimization in graphs; characterization of connected subgraphs.

\section{Resumo}

Apresentamos modelos lineares inteiros com um número polinomial de variáveis e restrições para problemas de otimização combinatória em grafos: ciclos elementares ótimos, caminhos elementares ótimos e problemas em árvores ótimas.

Palavras-chave: modelos de problemas de otimização combinatória; otimização em grafos; caracterização de subgrafos conexos. 


\section{Introduction and Definitions}

Sometimes we have to solve small and median size combinatorial optimization problems using commercial codes of mixed linear integer programming as CPLEX, LINDO, OSL, XPRESS, etc. The use of branch and cut techniques to solve these problems needs a hard work of implementation. For the traveling salesman problem (TSP) and the Steiner tree problem (STP) in graphs one uses mathematical models with an exponential number of constraints to avoid subtours, these constraints are implicitly considered. The aim of this paper is to present 0-1 mixed linear programs with a polynomial number of variables and constraints to solve some combinatorial optimization problems using commercial codes.

When we solve integer linear programming by column generation techniques, we have to generate paths, cycles and trees with additional constraints in small graphs, 40 to 60 nodes, 120 to 180 edges, at each iteration. For this we can use a commercial code to solve the master problem and the same code to solve the optimization problem which generates at each iteration a new column.

We define our notation as follows.

Let $G=(V, E)$ be a connected graph, where $V=\{1,2,3, \ldots, n\}$ is the set of nodes or vertices and $E$ the set of edges. Let $G_{d}=(V, A)$ be a directed graph derived from $G$, where $A=\{(i, j),(j, i) \mid$ $\{i, j\} \in E\}$, that is, each edge $u=\{i, j\} \in E$ is associated with two $\operatorname{arcs}(i, j)$ and $(j, i) \in A$. We define two news graphs: $G^{0}=\left(V_{0}, E_{0}\right)$, and $G_{d}^{0}=\left(V_{0}, A_{0}\right)$ where $V_{0}=V \cup\{0\}$, $E_{0}=E \cup\{\{0, j\} \mid j \in V\}, A_{0} A \cup\{(0, j) \mid j \in V\}$.

Let $x=\left(x_{i}\right)_{i \in V} \in\{0,1\}^{|V|}, y=\left(y_{u}\right)_{u \in E_{0}} \in\{0,1\}^{\left|E_{0}\right|}$ two 0 - 1 vectors, and $z_{i j}^{k} \geq 0,(i, j) \in A_{0}$, $k \in V^{\prime}$ where $V^{\prime}$ is a subset of $V$, and $z_{i j}^{k}$ is a real flow in the $\operatorname{arc}(i, j) \in A_{0}$ having $O$ as source and $k$ as terminal. We consider $E(i)$ the set of edges $u \in E$ such that an endpoint is $i, \Gamma^{+}(i)=\left\{j \mid(i, j) \in A_{0}\right\}$ and $\Gamma^{-}(i)=\left\{j \mid(j, i) \in A_{0}\right\}, m=|E|$ and $n=|V|$.

All combinatorial optimization problems that will be treated in this paper are associated with connected subgraphs of $G$. In the section 2 we present a nonsimultaneous flow formulation based on Claus \& Maculan (1983), Beasley (1994), Wong (1984), Guyard (1985), Maculan (1986), Maculan (1987) and Maculan, Arpin \& Nguyen (1988) for the Steiner tree problem in graphs and in Claus (1984) and Figueiredo \& Maculan (2000) for the Travelling Salesman Problem (TSP) to guarantee that a subgraph has to be connected. We characterize all elementary cycles in a graph in section 3, and a particular model is developed for Hamiltonian cycles. In section 4 we propose a formulation to characterize all elementary paths in a graph. The characterization of subtrees with a fixed number of nodes in a graph is shown in section 5 . In section 6 we associate with each type of combinatorial optimization problem an objective function whose minimum has to be searched; the minimum Steiner tree problem in a graph is also formulated by a particular nonsimultaneous flow problem in this section. All theorems presented in this paper are easy to be proved. 


\section{Modeling a connected subgraph of $\mathbf{G}$}

The 0 - 1 vectors $x=\left(x_{i}\right)_{i \in V}$ and $y=\left(y_{u}\right)_{u \in E}$ defined above are associated with a subgraph of $G$ as follows: if $x_{i}=1$ then the node $i$ belongs to the subgraph, $x_{i}=0$ otherwise; if $y_{u}=1$ then the edge $u$ belongs to the subgraph, $y_{u}=0$ otherwise. These vectors will characterize a subgraph of $G$.

The dummy node 0 is included to be a source node for a nonsimultaneous flow problem. Our idea is the use of the nonsimultaneous flow problem to obtain connected graphs as follows.

$$
\begin{gathered}
\sum_{j \in \Gamma^{+}(0)} z_{0 j}^{k}-x_{k}=0, k \in V, \\
\sum_{j \in \Gamma^{+}(i)} z_{i j}^{k}-\sum_{j \in \Gamma^{-}(i)} z_{j i}^{k}=0, i \in V-\{k\}, k \in V, \\
\sum_{j \in \Gamma^{+}(k)} z_{k j}^{k}-\sum_{j \in \Gamma^{-}(k)} z_{j k}^{k}+x_{k}=0, k \in V, \\
z_{i j}^{k} \leq y_{i j} \text { and } z_{j i}^{k} \leq y_{i j},\{i, j\} \in E_{0}, k \in V . \\
y_{i j} \leq x_{i} \text { and } y_{i j} \leq x_{j},\{i, j\} \in E, \\
\sum_{j \in V} y_{0 j}=1 \\
z_{i j}^{k} \geq 0,(i, j) \in A_{0}, k \in V \\
y_{i j} \in\{0,1\},\{i, j\} \in E_{0}, \text { and } x_{k} \in\{0,1\}, k \in V .
\end{gathered}
$$

Theorem 1: All vectors $x$ and $y$ satisfying (1-8) are associated with connected subgraphs of $G$.

We consider that the subgraph associated with $x_{i}=0$, for all $\in V$, is a connected graph. If a connected subgraph has to have at least 2 nodes, then we include a new constraint as follows.

$$
\sum_{u \in E} y_{u} \geq 1
$$

or

$$
\sum_{i \in Z} x_{i} \geq 2
$$

Number of variables and constraints for the model defined by (1-8): 
Type of variables (connected subgraphs)

$$
\begin{gathered}
z_{i j}^{k} \\
y_{u} \\
y_{0 j} \\
x_{i} \\
\text { Total }
\end{gathered}
$$

Type of constraints (connected subgraphs)

(1) - (3)

total

Number
$2 m n+n^{2}$
$m$
$n$
$n$
$2 m n+n^{2}+m+2 n$

$$
\begin{gathered}
\text { Number } \\
n^{2}+n \\
4 m n+2 n^{2} \\
2 m \\
1 \\
2 m n+n^{2} \\
m+2 n \\
6 m n+4 n^{2}+3 m+3 n+1
\end{gathered}
$$

\section{Elementary Cycles}

We introduce new constraints which guarantee that each node in the subgraph has degree 2.

$$
\sum_{u \in E(i)} y_{u}=2 x_{i}, \quad i \in V
$$

Constraints in (5) will be replaced by (11).

Number of variables and constraints for the this last model:

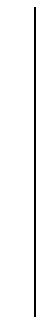

Type of variables (elementary cycles)

$$
\begin{gathered}
z_{i j}^{k} \\
y_{u} \\
y_{0 j} \\
x_{i} \\
\text { total }
\end{gathered}
$$

Type of constraints (elementary cycles)

$$
\text { (1) - (3) }
$$

total

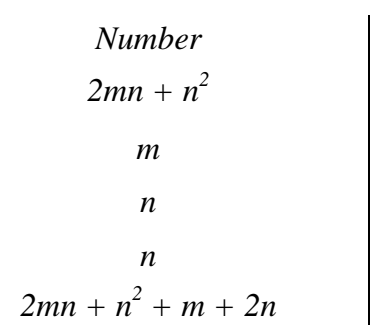

$$
\begin{gathered}
\text { Number } \\
n^{2}+n \\
4 m n+2 n^{2} \\
n \\
1 \\
2 m n+n^{2} \\
m+2 n \\
6 m n+4 n^{2}+m+4 n+1
\end{gathered}
$$


Theorem 2: All vectors $x$ and $y$ satisfying (1-4) and (6-11) are associated with elementary cycles of $G$.

The formulation presented in theorem 2 can be used to describe all $x$ and $y$ associated with Hamiltonian cycles, for this we put $x_{i}=1$, for all $i \in V$. In this case we will be able to propose another formulation using only $G$ and $G_{d}$, it is not necessary to include the 0 dummy node. We choose any node in $V$ to be the source node, we will take node 1 to be this source. Thus we present a new model as follows, where $\Gamma^{+}(i)=\{j \mid(i, j) \in A\}$ and $\Gamma^{-}(i)=\{j \mid(j, i) \in A\}$.

$$
\begin{gathered}
\sum_{j \in \Gamma^{+}(1)} z_{1 j}^{k}-\sum_{j \in \Gamma^{-}(1)} z_{j 1}^{k}=1, \quad k \in V-\{1\}, \\
\sum_{j \in \Gamma^{+}(i)} z_{i j}^{k}-\sum_{j \in \Gamma^{-}(i)} z_{j i}^{k}=0, \quad i \in V-\{1, k\}, \quad k \in V-\{1\}, \\
\sum_{j \in \Gamma^{+}(k)} z_{k j}^{k}-\sum_{j \in \Gamma^{-}(k)} z_{j k}^{k}=-1, \quad k \in V-\{1\}, \\
z_{i j}^{k} \leq y_{i j} \text { and } z_{j i}^{k} \leq y_{i j},\{i, j\} \in E, k \in V-\{1\}, \\
\sum_{u \in E(i)} y_{u}=2, i \in V, \\
z_{i j}^{k} \geq 0,(i, j) \in A, k \in V-\{1\}, \\
y_{i j} \in\{0,1\},\{i, j\} \in E .
\end{gathered}
$$

Theorem 3: Each vector y satisfying (12 - 18) is associated with a Hamiltonian cycle of G.

Number of variables and constraints for the Hamiltonian cycle model:

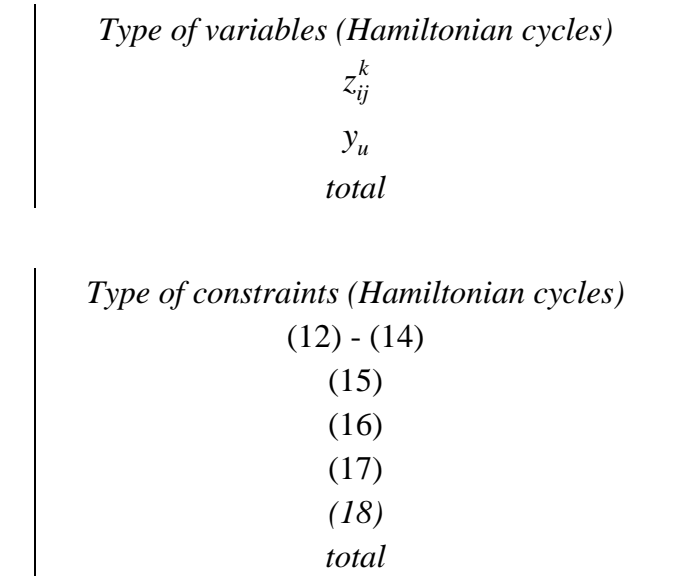

Number
$2 m n$
$m$
$2 m n+m$

Number
$n^{2}-n$
$2 m n-2 m$
$n$
$2 m n$
$m$
$4 m n+n^{2}-m$




\section{Elementary Paths}

Given two different nodes $i \in V$, and $j \in V$ we would like to characterize all elementary paths in $G$ between $i$ and $j$. For this $x_{i}=x_{j}=1$, and replace in the connected subgraph model constrains in (5) by

$$
\begin{gathered}
\sum_{u \in E(l)} y_{u}=2 x_{l}, l \in V-\{i, j\} \\
\sum_{u \in E(l)} y_{u}=1, l \in\{i, j\},
\end{gathered}
$$

Theorem 4: All vectors $x$ and $y$ satisfying $(1-4),(6-8)$ and $(19-20)$ are associated with elementary paths of $G$ between nodes $i$ and $j$.

\section{Trees}

First of all we characterize all subtrees in $G$ with a given number $p \leq n$ of nodes, see Fischetti et al. (1984). For this we have to consider new constraints as follows.

$$
\begin{gathered}
\sum_{i \in V} x_{i}=p, \\
\sum_{u \in E} y_{u}=p-1,
\end{gathered}
$$

Theorem 5: All vectors $x$ and $y$ satisfying $(1-8)$ and $(21-22)$ are associated with subtrees with $p \leq n$ nodes of $G$.

For characterizing all spanning trees of $G$ we just put $p=n$. In this case we can propose a new model, as we did for Hamiltonian cycles in $G$. Thus we propose the following mathematical model.

$$
\begin{gathered}
\sum_{j \in \Gamma^{+}(1)} z_{1 j}^{k}-\sum_{j \in \Gamma^{-}(1)} z_{j 1}^{k}=1, \quad k \in V-\{1\}, \\
\sum_{j \in \Gamma^{+}(i)} z_{i j}^{k}-\sum_{j \in \Gamma^{-}(i)} z_{j i}^{k}=0, \quad i \in V-\{1, k\}, \quad k \in V-\{1\}, \\
\sum_{j \in \Gamma^{+}(k)} z_{k j}^{k}-\sum_{j \in \Gamma^{-}(k)} z_{j k}^{k}=-1, \quad k \in V-\{1\}, \\
z_{i j}^{k} \leq y_{i j} \text { and } z_{j i}^{k} \leq y_{i j}, \quad\{i, j\} \in E, \quad k \in V-\{1\}, \\
\sum_{u \in E} y_{u}=n-1, \\
z_{i j}^{k} \geq 0, \quad(i, j) \in A, \quad k \in V-\{1\}, \\
y_{i j} \in\{0,1\},\{i, j\} \in E .
\end{gathered}
$$

Theorem 6: Each vector y satisfying (23 - 29) is associated with a spanning tree of G. 


\section{Combinatorial Optimization}

We associate real numbers (costs, weights, etc.) $c_{i}$ with nodes $i \in V$, and $w_{u}$ (costs, distances, weights, etc.) with edges $u \in E$. Thus we are concerned to deal with an objective function $f$ as follows.

$$
f(x, y)=\sum_{i \in V} c_{i} x_{i}+\sum_{u \in E} w_{u} y_{u} .
$$

That is $f:\{0,1\}^{|V|} \times\{0,1\}^{|E|} \rightarrow \Re$. We would like to find a connected subgraph of $G$ which minimizes (30). The connected subgraph optimization problem (CSP) bellow will give an optimal solution.

$$
\begin{gathered}
(C S P): \text { minimize } \sum_{i \in V} c_{i} x_{i}+\sum_{u \in E} w_{u} y_{u}, \\
\text { subject to } \\
\text { constraints }(1-8)
\end{gathered}
$$

The traveling salesman problem (TSP) is to obtain a Hamiltonian cycle of $G$ such that this cycle minimizes an objective function which represents the total distance of Hamiltonian cycles. Thus

$$
\begin{gathered}
(T S P): \text { minimize } \sum_{u \in E} w_{u} y_{u}, \\
\text { subject to } \\
\text { constraints }(12-18) .
\end{gathered}
$$

Where $w_{u}, u=\{i, j\} \in E$, is the distance between the extremes $i$ and $j$ of the edge $u$.

If $G$ is not a Hamiltonian graph, we will have no solution.

This TSP-formulation was used in Figueiredo \& Maculan (2000) to solve small and median size TSP-problems.

We can define the minimum Steiner tree problem (MSTP) in $G=(V, E)$ as follows. Given $\bar{V}$ a subset of $V$. With each edge $u \in E$ a cost $c_{u} \geq 0$ is associated. Find a minimum total cost subtree of $G$ that spans $\bar{V}$. The following mathematical programming solves the (MSTP), where we suppose that node $1 \in \bar{V}$.

$$
(M S T P): \text { minimize } \sum_{u \in E} c_{u} y_{u},
$$

subject to:

$$
\begin{gathered}
\sum_{j \in \Gamma^{+}(1)} z_{1 j}^{k}-\sum_{j \in \Gamma^{-}(1)} z_{j 1}^{k}=1, \quad k \in \bar{V}-\{1\}, \\
\sum_{j \in \Gamma^{+}(i)} z_{i j}^{k}-\sum_{j \in \Gamma^{-}(i)} z_{j i}^{k}=0, \quad i \in V-\{1, k\}, \quad k \in \bar{V}-\{1\}, \\
\sum_{j \in \Gamma^{+}(k)} z_{k j}^{k}-\sum_{j \in \Gamma^{-}(k)} z_{j k}^{k}=-1, \quad k \in \bar{V}-\{1\}, \\
z_{i j}^{k} \leq y_{i j} \text { and } z_{j i}^{k} \leq y_{i j}, \quad\{i, j\} \in E, \quad k \in \bar{V}-\{1\},
\end{gathered}
$$




$$
\begin{gathered}
z_{i j}^{k} \geq 0, \quad(i, j) \in A, \quad k \in \bar{V}-\{1\}, \\
y_{i j} \in\{0,1\},\{i, j\} \in E .
\end{gathered}
$$

The linear programming relaxation of this MSTP-formulation has given very good lower bounds for large scale Steiner problems in rectangular grid graphs, see Bahiense, Maculan \& Sagastizábal (2002).

\section{Conclusions}

Mathematical programming formulations for small and median size combinatorial optimization problems in graphs developed in this paper can give us optimal solutions using standard mixed integer programming softwares. Well-known modeling languages as AMPL, GAMS, LINGO, MP-MODEL (XPRESS) would be used to create the data of a mixed integer programming which will be solved by standard softwares.

\section{References}

(1) Bahiense, L.; Maculan, N. \& Sagastizábal, C. (2002). The volume algorithm revisited: relation with bundle methods. Mathematical Programming, 94, 41-69.

(2) Beasley, J.E. (1994). An algorithm for the Steiner problem in graphs. NETWORKS, 4, $1-1$.

(3) Claus, A. (1984). A new formulation for the travelling salesman problem. SIAM Journal of Algebraic and Discrete Methods, 5, 21-25.

(4) Claus, A. \& Maculan, N. (1983). Une nouvelle formulation du problème de Steiner sur un graphe. Publication 280, Centre de Recherche sur les Transports, Université de Montréal, Canada.

(5) Figueiredo, R.M.V. \& Maculan, N. (2000). O problema do caixeiro viajante assimétrico: limites inferiores e um novo algoritmo de separação. Investigação Operacional, 20, 127-146.

(6) Fischetti, M.; Hamacher, H.W.; Jornsten, K. \& Maffioli, F. (1984). Weighted K-cardinality trees: Complexity and polyhedral structure. NETWORKS, 14, 147-159.

(7) Guyard, L. (1985). Le problème de l'arbre de Steiner: modélisation par la programation linéaire et résolution par des techniques de décomposition. Thèse de Docteur Ingénieur, Ecole Nationale Supérieure des Télécommunications, Paris, France, 1985.

(8) Maculan, N. (1986). A new linear programming problem for the shortest s-directed spanning tree problem. Journal of Combinatorics, Information and System Science, 11, 53-56.

(9) Maculan, N. (1987). Steiner problem in graphs. Annals of Discrete Mathematics, 31, 185-212.

(10) Maculan, N.; Arpin, D. \& Nguyen, S. (1988). Le problème de Steiner sur un graphe orienté: formulations et relaxations. Computational and Applied Mathematics, 7, 1988, 109-118.

(11) Wong, R.T. (1984). A dual ascent approach to Steiner tree problem on a directed graph. Mathematical Programming, 28, 271-287. 